\title{
Relativity Isoframes-A Useful and Potentially Unifying Conceptual Framework
}

\author{
Eugene Terry Tatum \\ Independent Researcher, Bowling Green, KY, USA \\ Email: ett@twc.com
}

How to cite this paper: Tatum, E.T. (2021) Relativity Isoframes-A Useful and Potentially Unifying Conceptual Framework. Journal of Modern Physics, 12, 731-738. https://doi.org/10.4236/jmp.2021.126046

Received: March 8, 2021

Accepted: May 5, 2021

Published: May 8, 2021

Copyright (c) 2021 by author(s) and Scientific Research Publishing Inc. This work is licensed under the Creative Commons Attribution International License (CC BY 4.0).

http://creativecommons.org/licenses/by/4.0/ (cc) (i) Open Access

\begin{abstract}
This brief note introduces the conceptual framework of special and general relativity isoclocks and isoframes. Isoclocks and isoframes, as defined herein, can be used to create geometrical maps of space and time ("space-time") with and without matter embedded. They are useful for having a mental picture of space-time relationships without having to picture 4-dimensional manifolds, which very few students and scientists are able to do. With the aid of the optical lensing definition of curvature as inverse radius, a new gravitational force equation is derived, which also incorporates Einstein's mass/energy relation in the $m_{x}$ term. Thus, one may see how it is that gravitational force correlates with its time-embedded curvature-squared $\left(C_{x}^{2}\right)$ space in a more accurate formulation than could be envisioned by Newton. This becomes more apparent in high gamma fields, such as found near a black hole horizon. It is hoped that probability theories, such as quantum field theories in curved space-time, might be adaptable to the general relativity isoframe concept introduced herein.
\end{abstract}

\section{Keywords}

Isoframe, Isoclock, General Relativity, Special Relativity, Space-Time, Black Holes, Krogdahl, Unification

\section{Introduction and Background}

Kip Thorne, who shared the 2017 Nobel Prize in physics for his theoretical work on black holes, began his Black Holes \& Time Warps book [1] with thought experiments for three black holes of radically-different sizes. As Thorne pointed out on page 33 of his book, bizarre things should happen to radio signals received by observers as the transmitter approaches very close to the horizon of a black hole of any size. The signal frequency plummets rapidly towards zero, and 
the signal disappears entirely at the horizon (as the wavelength becomes infinite and the wave energy becomes zero). For the outside observer at a fixed radius from the horizon, it is as if a clock (or radio transmitter of a given frequency) becomes frozen in time relative to that observer. For observers falling into a black hole, it is an entirely different perspective (i.e., reference frame), but that is not the focus of this paper.

Albert Einstein's genius had much to do with his ability to find the right thought experiment and reference frame in which to simplify and understand a complex problem at a fundamental level [2]. With special relativity, his concept of the proper time clock of an observer in a chosen reference frame in comparison to the time clock of an observer in a different reference frame was of paramount importance. It was this clock comparison that mattered most to him, if one were to accept the embedded assumption of invariance of speed of light measurements.

In relativity theory, the proper time clock of a given reference frame (we'll call it $T_{o}$ ) can be compared to those of different reference frames. In fact, an ordering of all other reference frame clocks can be mapped with respect to the chosen clock of reference. One can utilize a subscript numbering system of the various clocks, such that clocks within the same reference frame can be numbered the same, and faster-ticking and slower-ticking clocks within different reference frames can be numbered higher or lower, respectively. Clocks keeping the same proper time are defined herein as "isoclocks," and their collective reference frame is defined herein as their "isoframe."

\section{Results: Isoframe Mappinig in Special and General Relativity}

Since a relativity isoframe is defined as one in which variously-positioned observers all keep the same proper time, their clocks can be considered to be perfectly synchronized at all times within their isoframe map.

\subsection{Special Relativity}

One can map out a special relativity Minkowskiian space-time isoframe in the following way (see Figure 1).

This is a 2-dimentional slice of rectilinear space-time (i.e., no curves in the latticework). For the purpose of this thought experiment, one can think of every point of line intersection as a fixed point with respect to an observer at the centrally-located $T_{o}$ point. Therefore, all such points of intersection can be considered to be in the same reference frame (isoframe) with respect to this $T_{o}$ clock, and to have identical $T_{o}$ clocks (isoclocks), one of which is shown. Anyone moving inertially in this Minkowskiian plane relative to any $T_{o}$ clock moves the same with respect to all $T_{o}$ clocks, and must have a non- $T_{o}$ clock of their own. One can readily envision the Minkowskiian space to be filled with many different non- $T_{o}$ clocks, depending upon their particular reference frame velocity with 


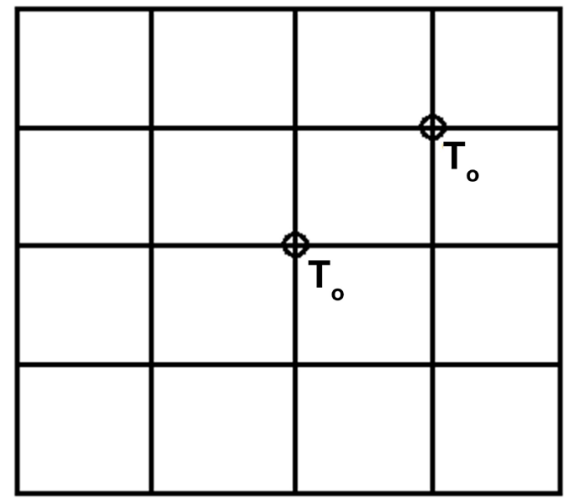

Figure 1. Special relativity isoframe (Minkowskiian space-time).

respect to the $T_{o}$ isoframe. So, the $T_{o}$ special relativity isoframe map of Figure 1 is one of stationary (in a relative sense) imaginary clocks in a field devoid of matter and its ever-present gravity.

\subsection{General Relativity}

Einstein's gravity theory, incorporating thought experiments with light beams and accelerating reference frames, necessitates that gravitationally-attractive matter positively curves space-time in a very specific way, according to a metric tensor (the "metric"). Without immersing oneself for years in this arcane branch of mathematics, one can nevertheless have a very good idea of how general relativity improves somewhat on the Newtonian concept of gravity in flat space and absolute time.

The key to this understanding is, once again, an isoframe mapping of identical time clocks, but within the gravity space surrounding a concentration of matter and/or energy. One must remember that matter and energy are two sides of the same coin, by $E=m c^{2}$. In contrast to the special relativity isoframe map of $T_{o}$ clocks fixed in position relative to a specified $T_{o}$ observer clock, the general relativity isoframe map is a curved manifold of different clocks at different distances (i.e., radii) within the gravity well surrounding a centralized focus of matter/ energy. See Figure 2.

As well-proven by clocks in satellite orbits around Earth, clocks farther from a center of gravity tick faster (hence the higher subscript numbers) than clocks deeper in a gravity well. Four such orbital clocks are shown in Figure 2 for comparison. Each orbital sphere surface (represented by a circle in the two-dimensional figure) can, therefore, be considered to be a gravity isoframe, as defined by its isoclock. The slowest clocks, in comparison to any of these orbital clocks, are those on Earth's surface, which, assuming continuance of the numbering sequence, would be labelled as $T_{o}$ in Figure 2 .

For the purposes of a general relativity thought experiment, let us consider a perfectly homogeneous and spherical extended body of matter which we will call a "planet." This planet has no angular momentum (i.e., it does not rotate) and all of its mass can be effectively treated as if it were concentrated at a point at the 


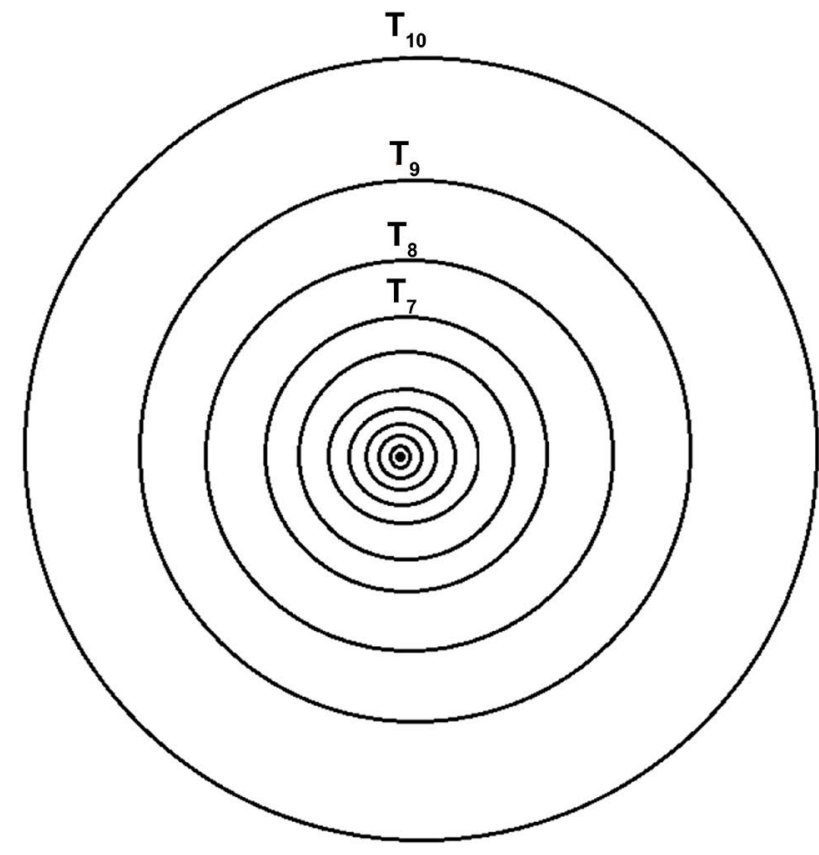

Figure 2. General relativity isoframes ( $T_{X}$ curved space-time).

geometric center of the sphere. Furthermore, this planet has no atmosphere and rests in a vacuum of infinite space with no other matter or energy.

We know from Newtonian mechanics that perfectly circular orbits centered on the planetary center can be achieved at any distance from the planetary center which is also beyond the planetary surface. We also know that a centripetal gravitating force of attraction on a body $(x)$ in a given circular orbit closely approximates

$$
F_{G x}=G M m_{x} r_{x}^{-2}
$$

wherein the usual Newtonian symbolic representation applies. Furthermore, each circular orbit $(x)$ can be defined by its curvature $C_{x}$ according to $r_{x}^{-1}$. This curvature definition is as useful for gravity in this simplified isoframe approach as it has been for the field of lens optics. Figure 3 replicates Figure 2, except that each orbital in the gravity well is now designated by its curvature $C_{x}$. The only important difference to keep in mind here is that a smaller curvature subscript $(x)$ in Figure 3 corresponds to a greater degree of curvature.

\section{Discussion}

One of the interesting features about this correlation between an $X$-orbital's curvature $C_{x}$ in space and its own proper time $T_{x}$ is that the square of its curvature $\left(C_{x}^{2}\right)$ correlates with gravitational force in proportion to $r_{x}^{-2}$. Thus,

$$
F_{G x}=G M m_{x} C_{x}^{2}
$$

Furthermore, while the $G M m_{x}$ term is a constant in the Newtonian theory, it can now actually be considered a variable according to $E=m c^{2}$. The rest massenergy of body $m_{x}$ on the planetary surface is fractionally less than the mass- 


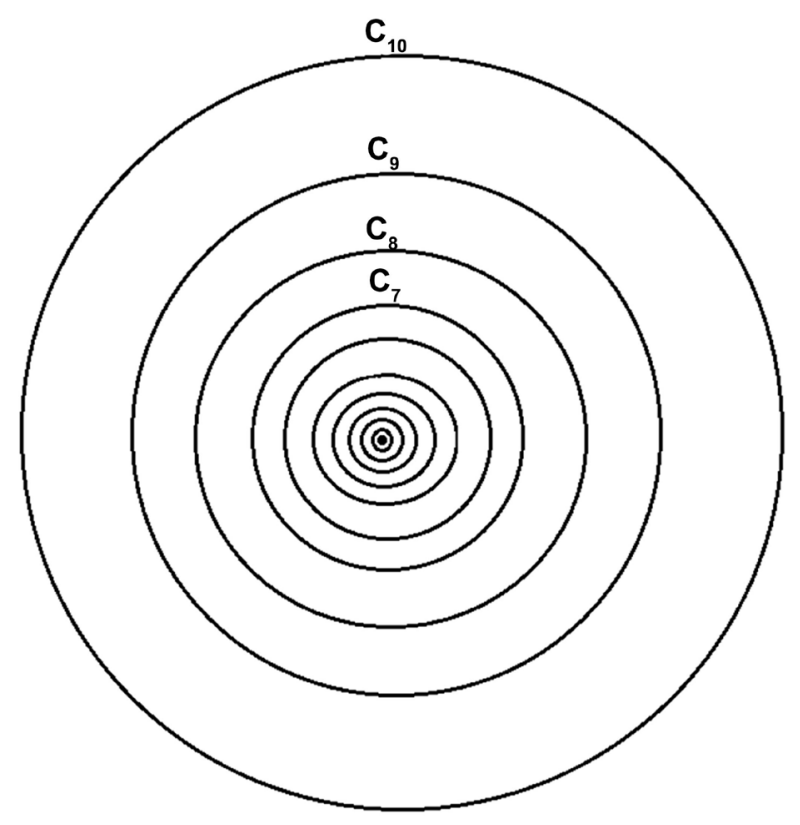

Figure 3. General relativity isoframes ( $C_{X}$ curved space-time).

energy of orbiting body $m_{x}$ at a certain height $h$ above the surface, according to $m \gamma h$ (gravitational potential energy) added to the rest mass-energy. Herein, we are using gravity field symbol $\gamma$ (acceleration due to gravity) in a generic sense, corresponding to a given height above a planet of mass $M$. The importance of now requiring the incorporation of $E=m c^{2}$ into the $m_{x}$ term of $G M m_{x} C_{x}^{2}$ is to show how it is that the centripetal gravitational force calculated by Newton always very slightly underestimates the general relativity force on orbiting bodies in relatively weak gravity fields. However, more importantly, it would be expected to greatly underestimate the general relativity force (including tidal forces) on orbiting bodies in strong (i.e., high energy density) gravity fields, such as those bordering black hole event horizons. Thus, Newton's theory can only be considered to be a very good approximation where gravitational energy density (and its associated space-time curvature) is relatively weak.

One of the reasons why $F_{G x}=G M m_{x} C_{x}^{2}$, when it incorporates $E=m c^{2}$ into the equation, appears to be a significantly better approximation of the true gravitational force than Newton's approximation is that Krogdahls incorporation of $E=m c^{2}$ into his own flat space-time cosmology formulation has already been shown to be remarkably accurate with respect to the canonical tests of any gravity theory competing with general relativity [3] [4].

Just as one can transform a square into a cube by multiplying by a measure of the 2-dimentional object (namely, its length), a squaring of the 2-dimensional circle curvature according to $C_{x}^{2}=r_{x}^{-2}$ can be considered to be a 3-dimensional representation of a $T_{x}$ orbital. In this way, a 2-dimensional spatial object with an embedded time clock corresponding to its curvature radius becomes a 3-dimensional spatial object with an embedded time clock corresponding to its curvature radius. Thus, the manifold of concentric spheres with different embedded isoc- 
locks becomes a conceptually useful model of 4-D space-time.

With respect to black holes, curved general relativity isoframes, with the third spatial dimension withheld for clarity, can be mapped around a perfectly spherical Schwarzschild black hole (see Figure 4).

In this figure, $T_{o}$ represents the frozen time clock of the black hole horizon, as perceived by a stationary or orbiting outside observer at any fixed radius outside the black hole horizon. It is often said that if one could instantaneously convert the sun into a Schwarzschild black hole of identical mass, the curved space-time of known planetary orbitals would look identical. The known planets would all be at such sufficient distances from the three-kilometer radius solar mass black hole horizon that we would not observe any appreciable difference in their orbital paths or periods.

The key difference in the adaptation of general relativity isoframes to black holes is what happens in close proximity (i.e., within several Schwarzschild radii) to the horizon. Here the gravitational field strength and tidal forces deviate significantly from Newtonian theory, and the $m \gamma h$ mass-energy contribution to $m_{x}$ becomes of paramount importance. At this point, by not incorporating $E=m c^{2}$ into $m_{x}$ Newtonian theory fails to be a close approximation of the extreme tidal forces and other phenomena occurring at orbital radii near a black hole horizon. Obviously, the frozen horizon clock $T_{o}$ in Figure 4 marks the boundary of the black hole isoframe map. Nothing certain or useful can be said about the interior of a black hole. And no one wants to be the free-falling observer to pass this horizon and explore the interior!

One wonders if the above general relativity isoframe conceptualization could be of use in terms of unification with quantum physics. Quantum field theories

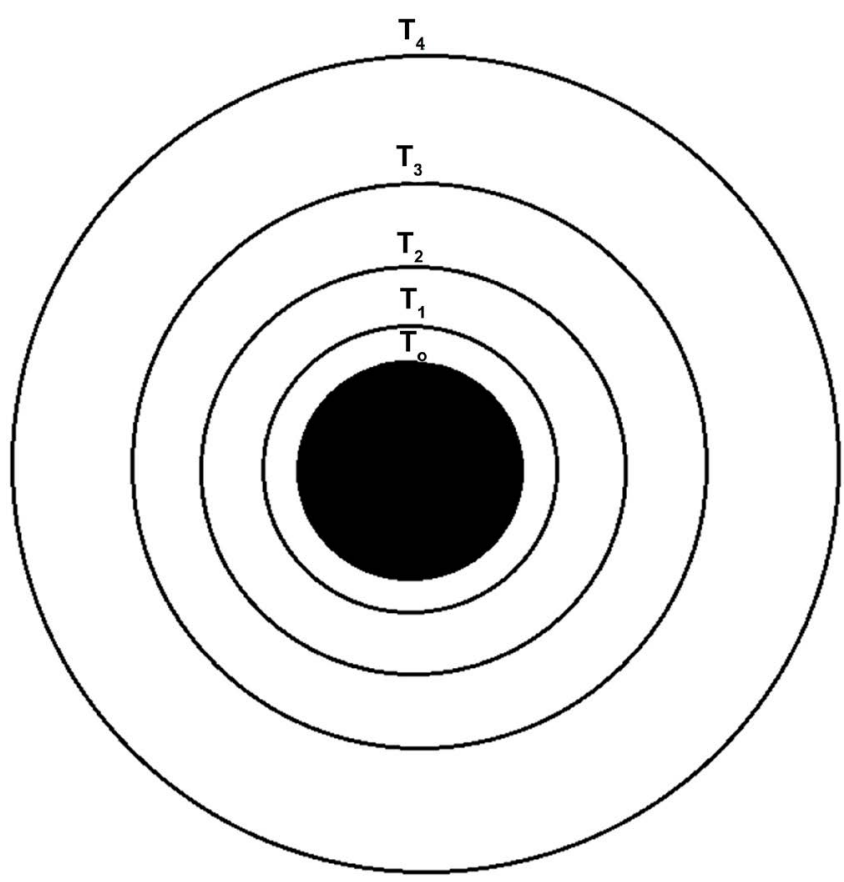

Figure 4. General relativity isoframes around a black hole. 
in curved space-time do, in fact, exist. These generally follow the local structure (i.e., the geometry) of space-time. Therefore, it is hoped that the conceptual framework presented herein may be useful in some way to quantum physicists working towards unification.

\section{Summary and Conclusions}

This paper has introduced readers to the definition and mapping of isoclocks and isoframes in special and general relativity. Particular attention has been paid to general relativity isoframe mapping around an ideal spherical body of matter/energy, according to the mapping of isoclocks and their isoframe orbitals. Application of the $C_{x}=r_{x}^{-1}$ curvature definition, borrowed from optical lensing, allows one to define and represent time-embedded orbital curvature in 2-dimensional space. Furthermore, squaring of curvature $\left(C_{x}^{2}=r_{x}^{-2}\right)$ allows one to define and represent time-embedded curvature in 3-dimensional space. And because of its time-embedded nature, this becomes a useful analogue of 4-dimensional space-time. The mapping of concentric time-embedded spheres of this type is conceptually useful for the majority of us who cannot otherwise picture 4-dimensional manifolds. Furthermore, the introduction of isoframe formula $F_{G X}=G M m_{x} C_{x}^{2}$, may allow one to see how it is that gravitational force not only correlates with $r_{x}^{-2}$, but also time-embedded curvature space (space-time). Finally, the author speculates that probability theories, such as quantum field theories in curved space-time, might be adaptable to the general relativity isoframe concept introduced herein.

\section{Data Availability}

Due to the purely theoretical nature of this undertaking, no new data were generated or analyzed in support of this research.

\section{Dedications and Acknowledgements}

This paper is dedicated to Dr. Wasley S. Krogdahl for his work on cosmology in flat space-time, and to Drs. Stephen Hawking and Roger Penrose for their groundbreaking work on black holes and cosmology. Dr. Tatum also thanks Dr. Rudolph Schild of the Harvard-Smithsonian Center for Astrophysics for his past support and encouragement.

\section{Conflicts of Interest}

The author declares no conflicts of interest regarding the publication of this paper.

\section{References}

[1] Thorne, K.S. (1994) Black Holes \& Time Warps. W.W. Norton \& Company, New York.

[2] Pais, A. (1982) Subtle is the Lord: The Science and the Life of Albert Einstein. Ox- 
ford University Press, Oxford.

[3] Krogdahl, W.S. (2006) Cosmology in Flat Space-Time. arXiv:gr-qc/0402016.

[4] Tatum, E.T. (2017) Journal of Modern Physics, 8, 2087-2095. https://doi.org/10.4236/jmp.2017.813127 